\title{
Second-generation antipsychotic and diabetes mellitus in children and adolescents
}

\author{
Carlo Ripoli, ${ }^{1}$ Anna Paola Pinna, ${ }^{1}$ Faustina Podda, ${ }^{1}$ Roberta Zanni, ${ }^{2}$ Maria Giada Tronci, ${ }^{2}$ \\ Anna Maria Nurchi1 \\ ${ }^{1}$ Unit of Diabetology-I Pediatric Section, Department of Surgical Sciences, University of Cagliari; ${ }^{2}$ Centre for \\ Pharmacological Therapy in Child and Adolescent Neuropsychiatry, Department of Neuroscience B.B. Brodie, \\ University of Cagliari, Cagliari, Italy
}

\begin{abstract}
Second generation antipsychotics (SGA) are used in children for the treatment of various psychiatric diseases, including pervasive developmental disorders. These drugs can cause metabolic effects as hyperglycemia and diabetes. A 16-year-old young-boy, diagnosed with autism, developed diabetes mellitus type 1 whilst he was on treatment with olanzapine (started 4 months before), clomipramine, valproic acid and lithium. The hypothesis of druginduced diabetes imposed olanzapine interruption and clozapine initiation. Insulin therapy was practiced, with progressive dosage reduction, until complete cessation of treatment after 13 months. Blood sugar and HbAlc levels remained stable for about a year and then increased again, requiring the introduction of metformin that improved glycemia. In children and adolescents assuming
\end{abstract}

Correspondence: Anna Paola Pinna, Unit of Diabetology-I Pediatric Section, Department of Surgical Sciences, University of Cagliari,

Cagliari, Italy.

Tel: +39-3488946860 - Fax: +39-07052963452.

E-mail: annapaola_pinna@yahoo.it

Key words: Diabetes mellitus; Second-generation antipsychotic; Children.

Conflict of interest: the authors declare no potential conflict of interest.

Human and animal rights: this article does not contain any studies with human or animal subjects performed by the any of the authors.

Informed consent: informed consent was obtained from all patients for being included in the study.

Contributions: all authors made substantial contributions to conception and design, acquisition of data, and analysis of data of this work.

Received for publication: 13 February 2017.

Revision received: 31 August 2017.

Accepted for publication: 15 November 2017.

This work is licensed under a Creative Commons Attribution

NonCommercial 4.0 License (CC BY-NC 4.0).

(C) Copyright C. Ripoli et al., 2017

Licensee PAGEPress, Italy

La Pediatria Medica e Chirurgica 2017; 39:149

doi:10.4081/pmc.2017.149
SGA serum glucose and lipid profile should always be assessed before therapy and then frequently monitored. Drug selection must consider family history and the individual risk. Molecule final choice remains equilibrium between efficacy and safety.

\section{Introduction}

Hyperglycemia and diabetes can be caused by multiple drugs. A 2010 pharmacovigilance study, analyzing 1219 drug notifications, showed that most frequently molecules responsible of blood sugar increase, according to ATC (Anatomical Therapeutic Chemical) classification, are antiretrovirals, steroids (prednisone and methylprednisolone), second generation neuroleptics, immunosuppressants (especially tacrolimus) and diuretics. ${ }^{1}$ They may act by increasing insulin resistance, by affecting insulin secretion, or both. Antipsychotic therapies for pediatric behavioral and affective disorders are increasingly used in children, in particular SGA are more effective and more tolerable than first-generation molecules, improving quality of life in billions of people worldwide. ${ }^{2}$

Recent controlled trials have documented the efficacy of some of these agents to treat severe aggression and self-injury characterizing autism in children and adolescents. Even if some doubleblind randomised placebo-controlled trials, open-label studies and case reports proved the usefulness of SGA in pervasive developmental disorders, few studies (and often in contrast) have pointed out their short and long-term safety. ${ }^{3}$ Despite the paucity of data, metabolic side effects, such as hyperglycemia, weight gain, hyperlipidemia and diabetes mellitus, seem to be more evident in children than in adults. Diabetes risk is increased by 2 to 3 -fold with use of antipsychotics.

In 2008 a classification of risk was proposed, showing that olanzapine and clozapine could, more frequently, cause weight gain, extrapyramidal side effects and metabolic changes compared to aripiprazole or ziprasidone. 4

A 2015 study, that examined 107551 SGA initiators, found that, compared with risperidone, ziprasidone and aripiprazole had a higher association with T2DM while quetiapine and olanzapine had lower risk than risperidone itself (Figure 1). ${ }^{5}$ This risk was documented early in the first year of therapy, and was elevated for up to 1 year after antipsychotic discontinuation. Moreover risk augmented significantly with increasing cumulative-dose. 6

The mechanism by which SGA can cause diabetes mellitus is twofold, both related to insulin resistance and to a reduction of insulin secretion.7,8 Insulin resistance can be associated with weight increase or can determine direct peripheral effects on adi- 
pose tissue, liver and muscle. Weight gain linked to antipsychotics occurs as a result of increased food intake rather than decreased energy expenditure. Increased energy intake is probably due to antagonistic effects of antipsychotic drugs on histamine 1 receptor and on serotonergic receptors. ${ }^{9}$

Recently, some polymorphisms, representing a genetic risk factor for antipsychotic treatment, because associated with weight gain, have been individuated. Examples are leptin (LEP) gene, fat mass and obesity-associated protein (FTO) gene, melanocortion-4receptor (MC4R) gene, serotonin 2C receptor (HTR2C) gene or methylenetetrahydrofolate reductase (MTHFR) gene. 10 A reduction of insulin secretion is, also, an important cause of abnormal glucose metabolism caused by antipsychotics. Beta cell disfunction is due to partial agonist activity of SGA on 5-HT2a and 5HT2c receptors, dopamine D2-like receptors and muscarinic M3 receptors, present in pancreatic $\beta$-cells. ${ }^{11}$ Olanzapine may induce apoptosis and inhibition of insulin secretion, blocking phosphorylation of the alfa subunit of eukaryotic initiation factor 2 . Subsequently proinsulin and insulin accumulate inside olanzapinetreated cells. Inhibition of protein synthesis and the reduction of insulin mRNA determine less burden of unfolded protein and then apoptosis of beta cells. ${ }^{12}$

Some children have subjective response to different antipsychotic drug, even if coming from the same class. Time of manifestation of diabetes and/or obesity after the beginning of therapy can range from 6 to 12 weeks up to 12 months depending upon the specific antipsychotic drug used and the individual genetic background. Often SGA-treated children maintain high weight and insulin resistance for long time, even after changes in life style and/or use of anti-diabetic drugs. ${ }^{13}$ The explanation of all these phenomena is multifactorial. Probably, in future, genetic technology will help us identifying children and adolescent who will respond best to a specific antipsychotic, causing as few adverse reactions as possible. ${ }^{2}$

\section{Case Report}

We present the case of a 16-year-old young-boy who was diagnosed with autism when he was three-years-old and that came to our observation because he developed diabetes mellitus whilst on treatment with olanzapine, clomipramine, valproic acid and lithi-

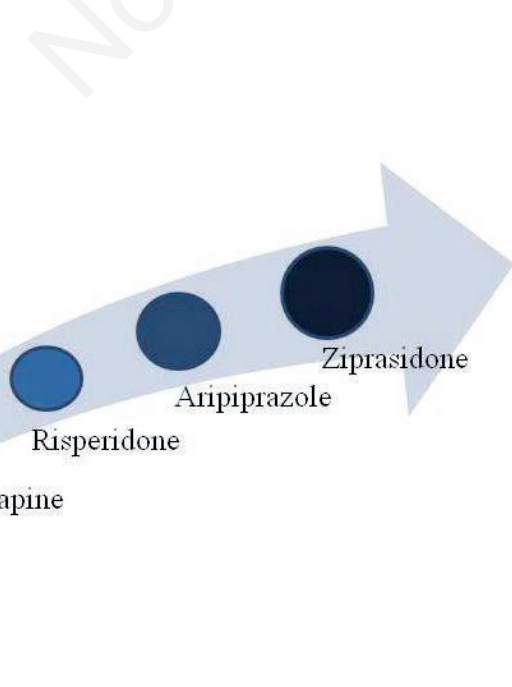

Figure 1. Atypical antipsychotics and diabetes mellitus associated risk. um. Before olanzapine he was treated with risperidone (from 10 years-old till 16 years-old), up to a dose of $3 \mathrm{mg} /$ day, without glycemic alterations. The presentation of progressive irritability and self-aggression led to the substitution of risperidone with quetiapine, without control of psychiatric symptoms. For this reason he began an oral therapy with olanzapine and, after 4 months of treatment, the child came to our emergency room with polyuria and polydipsia. He had a family history of type 2 diabetes, affecting paternal and maternal grandparents, while parents and 19 years-old brother had not glucose metabolism abnormalities and were normal weight. The young-boy was born at term, his birth weight was $3450 \mathrm{~g}$. At diagnosis anthropometric parameters were normal: BMI 22.8, glycemia $37 \mathrm{mmol} / \mathrm{L}$, Beta hydroxy butyrate $0.1 \mathrm{mmol} / \mathrm{L}$. Venous blood gas was normal. C-peptide baseline was $1.8 \mathrm{ng} / \mathrm{mL}$ and HbAlc $83 \mathrm{mmol} / \mathrm{mol}$ (9.7\%).

Islet cell antibodies (ICA), insulin autoantibodies (IAA), glutamic acid decarboxylase antibodies (GADA) and tyrosine phosphatase-related islet antigen 2 antibodies (IA-2) were negative. Moreover anti-endomysial antibodies (EMA), anti-tissue transglutaminase (anti-tTG) antibodies and thyroid function were regular. HLA was not diabetes type 1 predisposing. He had no signs of insulin resistance. Acanthosis nigricans was absent. Lipid panel and blood pressure were normal.

The hypothesis of drug-induced diabetes mellitus imposed olanzapine interruption and a close interaction with neuropsychiatric team that resulted in a gradual switching to clozapine, also because olanzapine was not useful in controlling the important irritability that compromised considerably his quality of life. Basalbolus insulin therapy was practiced with aspart and glargine.

Hyperglycemia was controlled by insulin doses of 0.6 $\mathrm{U} / \mathrm{kg} /$ day with a rapid resolution of clinical signs allowing a progressive reduction dosage until complete cessation of therapy after 13 months. HbAlc was $43 \mathrm{mmol} / \mathrm{mol}$ (6.1\%). It has been impossible to perform an oral glucose load following the normalization of blood sugar levels due to poor young-boy compliance.

Blood sugar and $\mathrm{HbA} 1 \mathrm{c}$ levels remained stable for about a year and then they increased again, requiring the introduction of metformin with a gradual normalization of glycemia. Considering that clozapine has resulted in a good compensation of the psychiatric symptoms for the first time from the beginning of treatment, we are evaluating with neuropsychiatric team the real cost-benefit ratio of a further modification of antipsychotic treatment. 14

\section{Discussion}

There is paucity of data concerning drug-induced diabetes in childhood. Diagnosis is based on medical history and excluding the other most common forms of diabetes mellitus. The young-boy we followed had no signs of type 1 diabetes mellitus, autoimmunity was negative and genetic HLA was no predisposing. Moreover his BMI, blood pressure, cholesterol values and the absence of insulin resistance signs make us excluding the presence of metabolic syndrome or type 2 diabetes mellitus.

Most frequent monogenic forms (MODY in particular) may be excluded by the absence of inheritance in parents. This case report is emblematic of individual difficulties and tailored-therapy need, trying to balance clinical benefits and the important adverse reactions.

There are only few cases of olanzapine-induced diabetes described in literature involving pediatric and adolescent population. Case report depicted relates with both type 1 (which can occur even with ketoacidosis) and type 2 diabetes. Time of onset from drug assumption is variable, and it has been studied above all in adult 
population where duration of antipsychotic therapy prior the onset of symptoms ranged from 4 days to 4 years (the majority occurred within 6 months after starting introduction of speculative drug).15-17

\section{Conclusions}

Unfortunately there are no elements emerging from clinical practice or from evidence-based medicine which can allow selecting children who will best respond to a specific antipsychotic agent. The only possibility is a strict follow up. For this reason in children and adolescents receiving second-generation antipsychotic drugs (specifically aripiprazole, olanzapine, clozapine) fasting blood glucose and lipid levels must be monitored frequently.

This attention is important above all in those children with obesity or a family history of diabetes mellitus in which every decision should be taken after considering the individual child presentation and family's preference. Follow up should be facilitated by simple flow chart that can help programming periodical exams. ${ }^{18}$ Clinical practice guidelines are available for monitoring adults and children who receive antipsychotic agents. ${ }^{19}$ The use of metformin, to prevent SGA metabolic effects, must also be considered in children. Metformin can attenuate weight gain and metabolic disorders in youths and it is generally well tolerated.

Finally, more trials about long-term efficacy and safety of second-generation antipsychotics are needed to individualize the decision on each patient's risk-benefit profile.

\section{References}

1. Chebane L, Tavassoli N, Bagheri H, Montastruc JL. Druginduced hyperglycemia: a study in the French pharmacovigilance database. Therapie 2010;65:447-58.

2. Briles JJ, Rosenberg DR, Brooks BA, et al. Review of the safety of second-generation antipsychotics: Are they really "atypically" safe for youth and adults? Prim Care Companion CNS Disord 2012;14:11r01298.

3. Allison DB, Mentore JL, Heo M, et al. Antipsychotic-induced weight gain: a comprehensive research synthesis. Am J Psychiatry 1999;156:1686-96.

4. Miller DD, Caroff SN, Davis SM, et al. Clinical Antipsychotic Trials of Intervention Effectiveness (CATIE) investigators. Extrapyramidal side-effects of antipsychotics in a randomised trial. Br J Psychiatry 2008;193:279-88.

5. Rubin D, Kreider A, Matone M, et al. Risk for incident diabetes mellitus following initiation of second-generation antipsychotics among medicaid-enrolled youths. JAMA Pediatr 2015;169:1-10.
6. Bobo WM, Cooper WO, Stein CM, et al. Antipsychotics and the risk of type 2 diabetes mellitus in children and youth. JAMA Psychiatry 2013;70:1067-75.

7. Kodama S, Horikawa C, Fujihara K, et al. Quantitative relationship between body weight gain in adulthood and incident type 2 diabetes: a meta-analysis. Obes Rev 2014;15:202-14.

8. Pramyothin P, Khaodhiar L. Type 2 Diabetes in children and adolescents on atypical antipsychotics. Curr Diab Rep. 2015;15:1-8.

9. Ballon JS, Pajvani U, Freyberg Z, et al. Molecular pathophysiology of metabolic effects of antipsychotic medications. Trends Endocrinol Metab 2014;25:593-600.

10. Shams TA, Muller DJ. Antipsychotic induced weight gain: genetics, epigenetics, and biomarkers reviewed. Curr Psych Rep 2014;16:473.

11. Starrenburg FC, Bogers JP. How can antipsychotics cause diabetes mellitus? Insights based on receptor-binding profiles, humoral factors and transporter proteins. J Assoc Eur Psychiatr 2009;24:164-70.

12. Ozasa R, Okada T, Nadanaka S, et al. The antipsychotic olanzapine induces apoptosis in insulin-secreting pancreatic beta cells by blocking PERK-mediated translational attenuation. Cell Struct Func 2013;38:183-95.

13. Rojo LE, Gaspar PA, Silva H, et al. Metabolic syndrome and obesity among users of second generation antipsychotics: A global challenge for modern psychopharmacology. Pharmacol Res 2015:101:74-85.

14. Toren P, Ratner S, Laor N, Weizman A. Benefit-risk assessment of atypical antipsychotics in the treatment of schizophrenia and comorbid disorders in children and adolescents. Drug Saf 2004;27:1135-56.

15. Bloch Y, Vardi O, Mendlovic S, et al. Hyperglycemia from olanzapine treatment in adolescents. J Child Adolesc Psychopharmacol 2003;13:97-102.

16. Courvoisie HE, Cooke DW, Riddle MA. Olanzapine-induced diabetes in a seven-year-old boy. J Child Adolesc Psycho-pharmacol 2004; 14:612-6.

17. Vuk A, Rojnic Kuzman M, Baretic M, Matovinovic Osvatic M. Diabetic ketoacidosis associated with antipsychotic drugs: case reports and a review of literature. Psychiatria Danubina 2017;29:121-35.

18. American Diabetes Association, American Psychiatric Association, American Association of Clinical Endocrinologists, North American Association for the Study of Obesity. Consensus development conference on antipsychotic drugs and obesity and diabetes. Diabetes Care 2004;27:596-601.

19. Pringsheim T, Panagiotopoulos C, Davidson J, et al. Evidencebased recommendations for monitoring safety of second generation antipsychotics in children and youth. J Canad Acad Child Adolesc Psychiatr 2011;20:218-33. 